\title{
An International Evaluation of Cognitive Reserve and Memory Changes in Early Old Age in 10 European Countries
}

\author{
Dorina Cadar ${ }^{a, b}$ Annie Robitaille ${ }^{c, d}$ Sean Clouston ${ }^{e}$ Scott M. Hofer ${ }^{d}$ \\ Andrea M. Piccinin ${ }^{d}$ Graciela Muniz-Terrera ${ }^{b, f}$ \\ ${ }^{a}$ Research Department of Epidemiology and Public Health, University College London (UCL), and ${ }^{b} \mathrm{MRC}$ Unit for \\ Lifelong Health and Ageing at UCL, London, UK; ${ }^{c}$ Faculty of Health Sciences, University of Ottawa, Ottawa, ON, and \\ ${ }^{\mathrm{d}}$ Department of Psychology, University of Victoria, Victoria, BC, Canada; e Department of Preventative Medicine, Stony \\ Brook University, New York, NY, USA; ${ }^{f}$ Centre for Dementia Prevention, University of Edinburgh, Edinburgh, UK
}

\section{Keywords}

Cognitive reserve $\cdot$ Memory $\cdot$ Education $\cdot$ Older adults .

Latent growth curve model

\begin{abstract}
Background: Cognitive reserve was postulated to explain individual differences in susceptibility to ageing, offering apparent protection to those with higher education. We investigated the association between education and change in memory in early old age. Methods: Immediate and delayed memory scores from over 10,000 individuals aged 65 years and older, from 10 countries of the Survey of Health, Ageing and Retirement in Europe, were modeled as a function of time in the study over an 8-year period, fitting independent latent growth models. Education was used as a marker of cognitive reserve and evaluated in association with memory performance and rate of change, while accounting for income, general health, smoking, body mass index, gender, and baseline age. Results: In most countries, more educated individuals performed better on both memory tests at baseline, compared to those less educated. However, education was not protective against faster decline, except for in Spain for both immediate and delayed recall (0.007 [SE $=0.003$ ] and $0.006[S E=0.002]$ ), and Switzerland for immediate recall
\end{abstract}

\section{KARGER}

E-Mail karger@karger.com www.karger.com/ned

\section{๑) 2017 The Author(s) \\ Published by S. Karger AG, Basel \\ Karge \\ Open access}

This article is licensed under the Creative Commons Attribution 4.0 International License (CC BY) (http://www.karger.com/Services/ OpenAccessLicense). Usage, derivative works and distribution are permitted provided that proper credit is given to the author and the original publisher.
(0.006 [SE $=0.003])$. Interestingly, highly educated Italian respondents had slightly faster declines in immediate recall $(-0.006$ [SE $=0.003])$. Conclusions: We found weak evidence of a protective effect of education on memory change in most European samples, although there was a positive association with memory performance at individuals' baseline assessment.

(c) 2017 The Author(s)

Published by S. Karger AG, Basel

\section{Introduction}

Preserved cognitive performance is a fundamental requisite of optimal ageing and an important determinant of the quality of life [1]. Cognitive reserve hypothesis was originally postulated, in part, to help explain individual differences in susceptibility to ageing or pathological cognitive decline. Cognitive reserve theory argues that people with higher cognitive reserve can perform and cope better with the neuropathological deterioration of the brain than individuals with lower reserve $[2,3]$. Educational attainment and adult socioeconomic status (SES) [4] are often considered proxies of cognitive reserve and used to provide empirical evidence for this hypothesis. Despite the pervasiveness of the cognitive reserve theory, 
the association between educational attainment and rate of cognitive decline has remained a topic of substantial interest. Some studies found no association between education and cognitive decline, while others have found a slower decline in individuals with higher education on specific subgroups or cognitive domains [5]. The inconsistencies in findings have been linked to methodological differences, population samples, diversity of cognitive tests used or the range of explanatory factors and covariates employed [6].

The purpose of this study was to assess the role of education as a marker of cognitive reserve on memory performance and change in individuals aged 65 years and older from 10 European countries part of the Survey of Health, Ageing and Retirement in Europe (SHARE), which employed the same research questions, methods, and covariates within each country. Due to the harmonised study design, SHARE provides an excellent opportunity to evaluate whether results replicate across the numerous countries involved in the SHARE study. To further reduce possible sources of heterogeneity that may emerge due to inconsistent analytical approaches, and to optimally evaluate the consistency of patterns of associations between cognitive reserve proxy and memory trajectories, we employed a coordinated analytical approach as proposed by Piccinin et al. $[7,8]$.

\section{Material and Methods}

\section{Data Sources}

SHARE is a multinational longitudinal study of 45,000 individuals born in 1954 or earlier (see www.share-project.org for details). Eleven countries contributed to the baseline data (2004) and were followed up biennially for further 4 waves. Participants selected for these analyses were 65 and older at baseline, who had completed the cognitive assessments on at least 2 separate occasions and had data on selected covariates (total sample $n=11,132$ ).

\section{Measures}

Cognitive evaluations were conducted in the first, second, fourth and fifth waves, in just 10 of 11 countries: Austria, Sweden, Germany, the Netherlands, Spain, Italy, France, Denmark, Switzerland, and Belgium, which were included in these analyses.

\section{Memory}

An immediate and a delayed 10 -word list recall were conducted as part of the Computer Assisted Personal Interviewing (CAPI). In the immediate recall, participants were asked to recall as many words as possible within one minute immediately after presenting them with a 10 -word list that has been read out. In the delayed recall, they were asked to recall as many words within one minute, after 5 min from the time of exposure, while they were presented with other information to prevent active rehearsal. Each word cor- rectly recalled scored 1 point (maximum score 10 for each test). Trained interviewers conducted face-to-face interviews using a laptop computer, on which the questionnaire was placed in the digital form. The generic CAPI questionnaire was administered uniformly across countries, using a similar computer-assisted interviewing system tool called "Blaise" that allowed each participating country to use the same interview format. The only differences in data collection procedure across countries were the native language used in the questionnaire and the local currency ${ }^{1}$ for reporting assets and income. For more details, see [9].

\section{Education and Covariates}

Information regarding educational achievement and all selected covariates was also collected as part of the CAPI interview. All participating countries had to answer the same set of questions and follow a similar set of procedures. Education represented the number of years of education completed by each participant. Income information referred to the gross value of annual household's income and was coded in deciles. General health was coded as excellent, very good, good, fair or poor. Other variables used were gender, body mass index (BMI), smoking, and age at the first wave of cognitive testing ("baseline").

To avoid potential biases due to differences between those who were educated in a country different from where they lived when interviewed, we included in the analytical sample only individuals who were born in the same country where they lived, after excluding 2,047 participants educated elsewhere (139 from Austria, 263 from Sweden, 308 from Germany, 194 from the Netherlands, 56 from Spain, 39 from Italy, 537 from France, 68 from Denmark, 170 from Switzerland, and 273 from Belgium).

\section{Statistical Analysis}

Immediate and delayed memory scores from each of the 10 countries were independently modeled as a function of years of study, fitting latent growth models (LGM). The level and rate of change were examined in association with education, income, health status, smoking, gender, BMI, and age at study entry. To ensure a coordinated analytical approach, we fitted random effects models to estimate the rate of change occurring linearly over time.

Continuous variables (age, education, and BMI) were centered at their respective country mean values. Household income percentiles were recorded such that the 50th percentile was the reference and treated as a continuous variable in the models. Information about self-perceived health was used to derive a binary indicator that took the value of 1 if respondents rated their health as excellent, very good, or good and 0 if fair or poor at the current time (baseline). Smoking habits were scored as 1 if participants smoked daily or 0 otherwise.

As a result of this coding and data harmonisation across countries, the intercept represents the average memory score at study entry and the slope of the rate of change in memory performance over a 8-year study period in an elderly man of 73-75 years of age, with 5.39-11.78 years of education and BMI of 25.0-27.4 according to each country mean values. His gross income is at the me-

\footnotetext{
${ }^{1}$ Despite most European countries using the EURO as their currency, some participants, particularly the oldest old, reported the use of previous currencies. In such cases, the interviewer converted the pre-Euro currency to Euro using a calculator on the laptop, as explained in Das et al. [9].
}

Cadar/Robitaille/Clouston/Hofer/ Piccinin/Muniz-Terrera 
dian of his country's income distribution and has never smoked, whose self-rated health was fair or poor.

Data analyses were conducted using MPlus (version 7.11) [10], and the figures were produced in STATA [11] and MATLAB [12].

\section{Results}

Demographics and cognitive scores at baseline and each follow-up wave are presented in Table 1 for all 10 participating countries (total $n=11,132$ ). The individual samples for each country included in these analyses are also described in Table 1.

\section{Performance at Study Entry and Decline Over Time}

Estimates and standard errors from the linear growth models for immediate (Table 2) and delayed (Table 3) memory performance at study entry and change over time are presented for each country included in these analyses.

At study entry, Germany, the Netherlands and Austria had the highest levels of performance on immediate recall $(4.54[\mathrm{SE}=0.10], 4.37[\mathrm{SE}=0.11]$, and $4.38[\mathrm{SE}=0.11]$, respectively), while Spain had the lowest performance $(2.93[\mathrm{SE}=0.11]$ ). For 6 out of the 10 countries (Austria, Denmark, Italy, The Netherlands, Sweden, and Germany), the change in immediate recall was minor but significant, while for Belgium, France, Spain, and Switzerland, it did not reach conventional statistical significance. Figure 1 illustrates the trajectories for both immediate and delayed memory change.

Similar to immediate recall, individuals from Germany $(2.80[\mathrm{SE}=0.11])$ and the Netherlands $(2.75[\mathrm{SE}=0.12])$ showed a better performance in delayed recall, while individuals from France $(1.93[\mathrm{SE}=0.10])$ and Spain $(1.42[\mathrm{SE}=0.09])$ had the lowest performance at study entry. Results regarding the rate of decline in memory were less consistent, reaching statistical significance only for 4 out of the 10 countries investigated (Denmark, Italy, The Netherlands, and Sweden).

\section{The Role of Education and Other Covariates on}

\section{Immediate Recall and Rate of Change (Table 2)}

In almost all countries, education was positively associated with immediate recall, such that more educated individuals performed better at study entry than those with fewer years of education. The only 2 exceptions were Austria and Switzerland. In most countries, education was not associated with the rate of change in immediate recall, except for Spain and Switzerland, where each additional year of education was found similarly associated with a slower rate of decline (0.007 [SE $=0.003]$ and $0.006[\mathrm{SE}=0.003])$; in

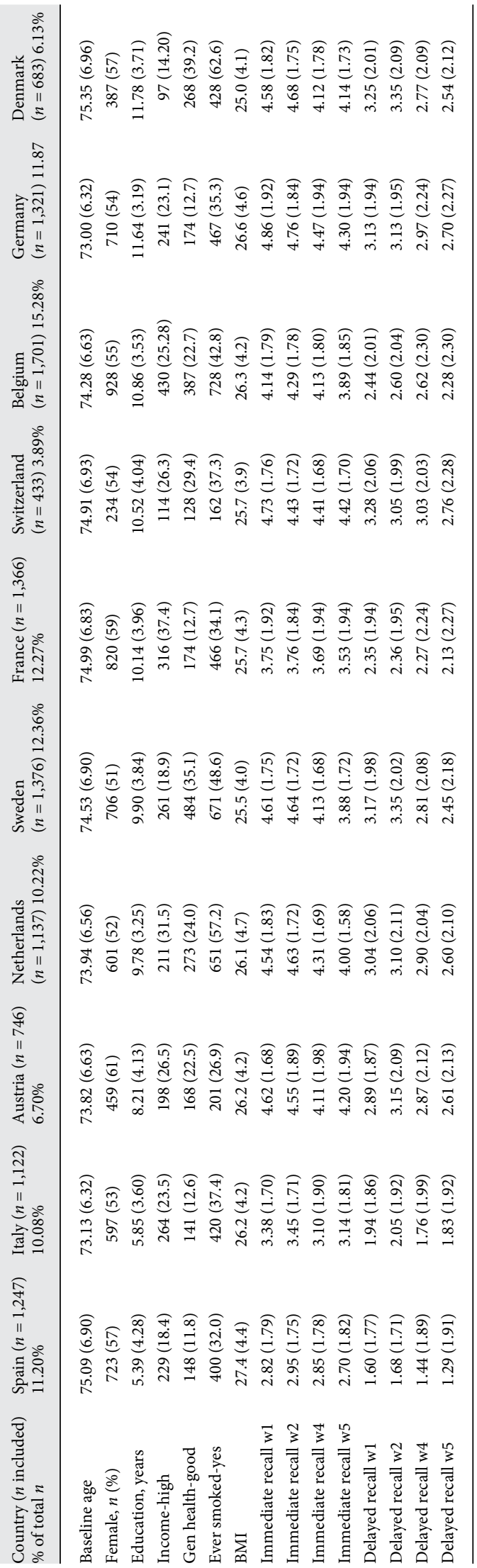


Table 2. Mean, SE of the estimates of the risk factors on random effects of the immediate memory recall mixed models

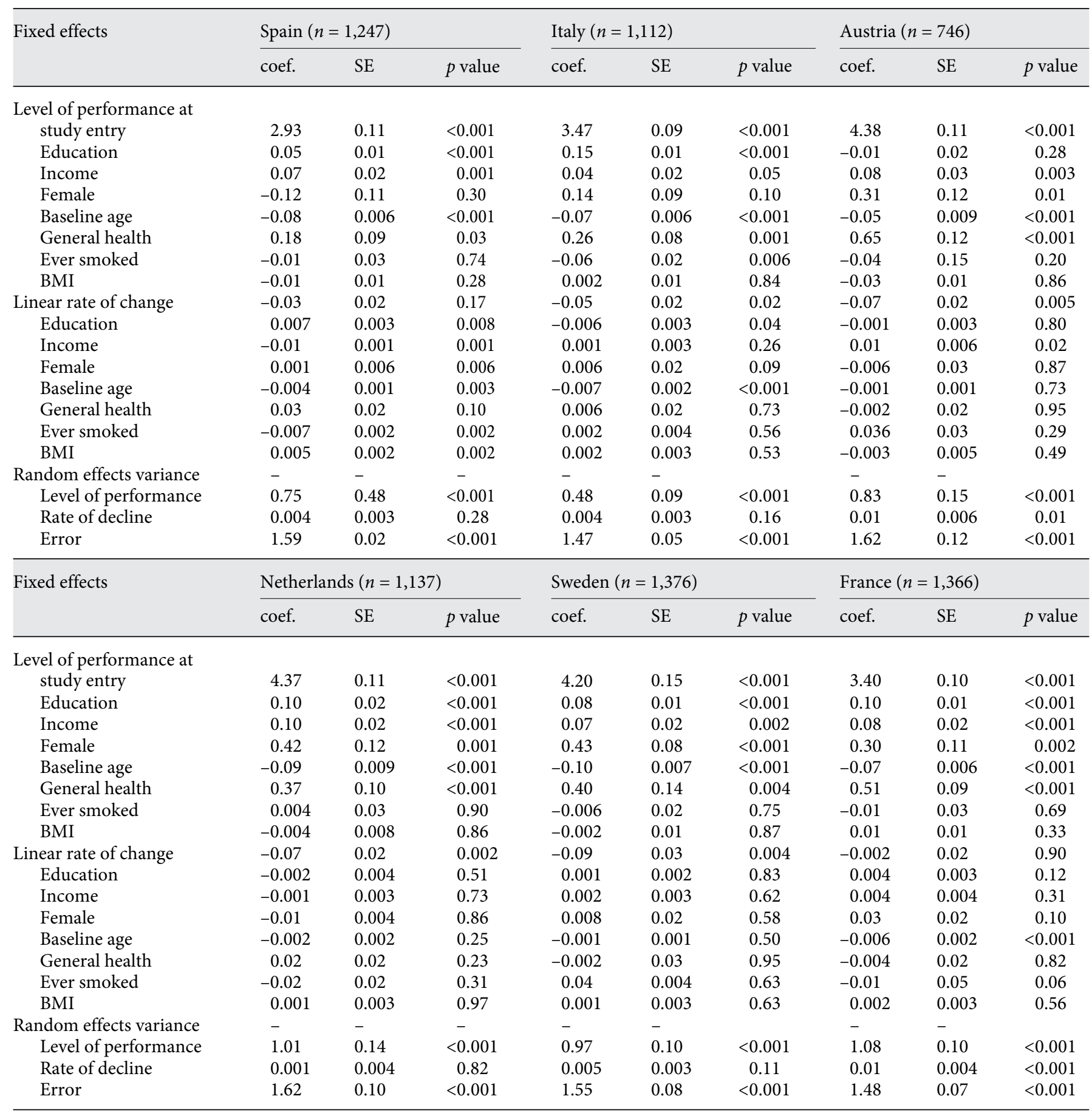

Italy, education showed an inverse association, predicting a slightly faster rate of decline $(-0.006$ [SE $=0.003])$.

All the individual country estimates of the effect of education on the immediate recall level at study entry and on the rate of change were meta-analysed (random effects models; Fig. 2a, c). The $I^{2}$ values obtained in the meta-analyses indicate high heterogeneity in between studies corresponding to the effect of education on immediate recall level estimates, but only moderate heterogeneity between studies on the estimates for the rate of change (slopes). 
Table 2. (continued)

\begin{tabular}{|c|c|c|c|c|c|c|c|c|c|}
\hline Fixed effects & coef. & SE & $p$ value & coef. & SE & $p$ value & coef. & SE & $p$ value \\
\hline Education & 0.02 & 0.02 & 0.35 & 0.12 & 0.01 & $<0.001$ & 0.12 & 0.02 & $<0.001$ \\
\hline Income & 0.11 & 0.03 & $<0.001$ & 0.04 & 0.02 & 0.02 & 0.07 & 0.02 & 0.003 \\
\hline General health & 0.48 & 0.20 & 0.02 & 0.26 & 0.08 & 0.002 & 0.54 & 0.09 & $<0.001$ \\
\hline Ever smoked & 0.03 & 0.04 & 0.37 & -0.03 & 0.02 & 0.25 & -0.02 & 0.02 & 0.33 \\
\hline BMI & -0.03 & 0.02 & 0.17 & 0.001 & 0.01 & 0.96 & 0.009 & 0.01 & 0.39 \\
\hline Linear rate of change & -0.03 & 0.05 & 0.57 & 0.007 & 0.02 & 0.70 & -0.07 & 0.03 & 0.01 \\
\hline Education & 0.006 & 0.003 & 0.04 & -0.003 & 0.002 & 0.15 & -0.001 & 0.003 & 0.64 \\
\hline Income & -0.009 & 0.006 & 0.13 & -0.003 & 0.003 & 0.26 & -0.01 & 0.005 & 0.008 \\
\hline BMI & 0.003 & 0.005 & 0.92 & 0.002 & 0.003 & 0.53 & -0.007 & 0.003 & 0.62 \\
\hline Random effects variance & - & - & - & - & - & & - & - & \\
\hline Level of performance & 0.93 & 0.17 & $<0.001$ & 1.21 & 0.09 & $<0.001$ & 0.74 & 0.15 & $<0.001$ \\
\hline Rate of decline & 0.001 & 0.005 & 0.95 & 0.02 & 0.003 & $<0.001$ & 0.007 & 0.006 & $<0.001$ \\
\hline Error & 1.50 & 0.13 & $<0.001$ & 1.34 & 0.05 & $<0.001$ & 1.62 & 0.12 & $<0.001$ \\
\hline \multirow[t]{2}{*}{ Fixed effects } & \multicolumn{3}{|c|}{ Denmark $(n=683)$} & & & & & & \\
\hline & coef. & SE & $p$ value & & & & & & \\
\hline \multicolumn{10}{|l|}{ Level of performance at } \\
\hline study entry & 4.10 & 0.14 & $<0.001$ & & & & & & \\
\hline Education & 0.09 & 0.02 & $<0.001$ & & & & & & \\
\hline Income & -0.006 & 0.005 & 0.34 & & & & & & \\
\hline Female & -0.01 & 0.02 & 0.61 & & & & & & \\
\hline Baseline age & -0.003 & 0.002 & 0.15 & & & & & & \\
\hline General health & -0.03 & 0.002 & 0.16 & & & & & & \\
\hline Ever smoked & 0.005 & 0.005 & 0.34 & & & & & & \\
\hline BMI & 0.002 & 0.004 & 0.53 & & & & & & \\
\hline Random effects variance & - & - & - & & & & & & \\
\hline Level of performance & 1.19 & 0.15 & $<0.001$ & & & & & & \\
\hline Rate of decline & 0.009 & 0.004 & $<0.001$ & & & & & & \\
\hline Error & 1.58 & 0.09 & $<0.001$ & & & & & & \\
\hline
\end{tabular}

Individuals with higher income performed better in immediate recall at study entry in all 10 countries investigated. However, despite having an advantage of better performance at the beginning of the survey, wealthier individuals were not protected against faster decline over time. The only exceptions were Austria, where wealthier individuals declined in their performance in immediate recall at a slower rate and Spain and Germany, where wealthier participants declined at a faster rate. 
Table 3. Mean, SE of the estimates of the risk factors on random effects of the delayed memory recall mixed models

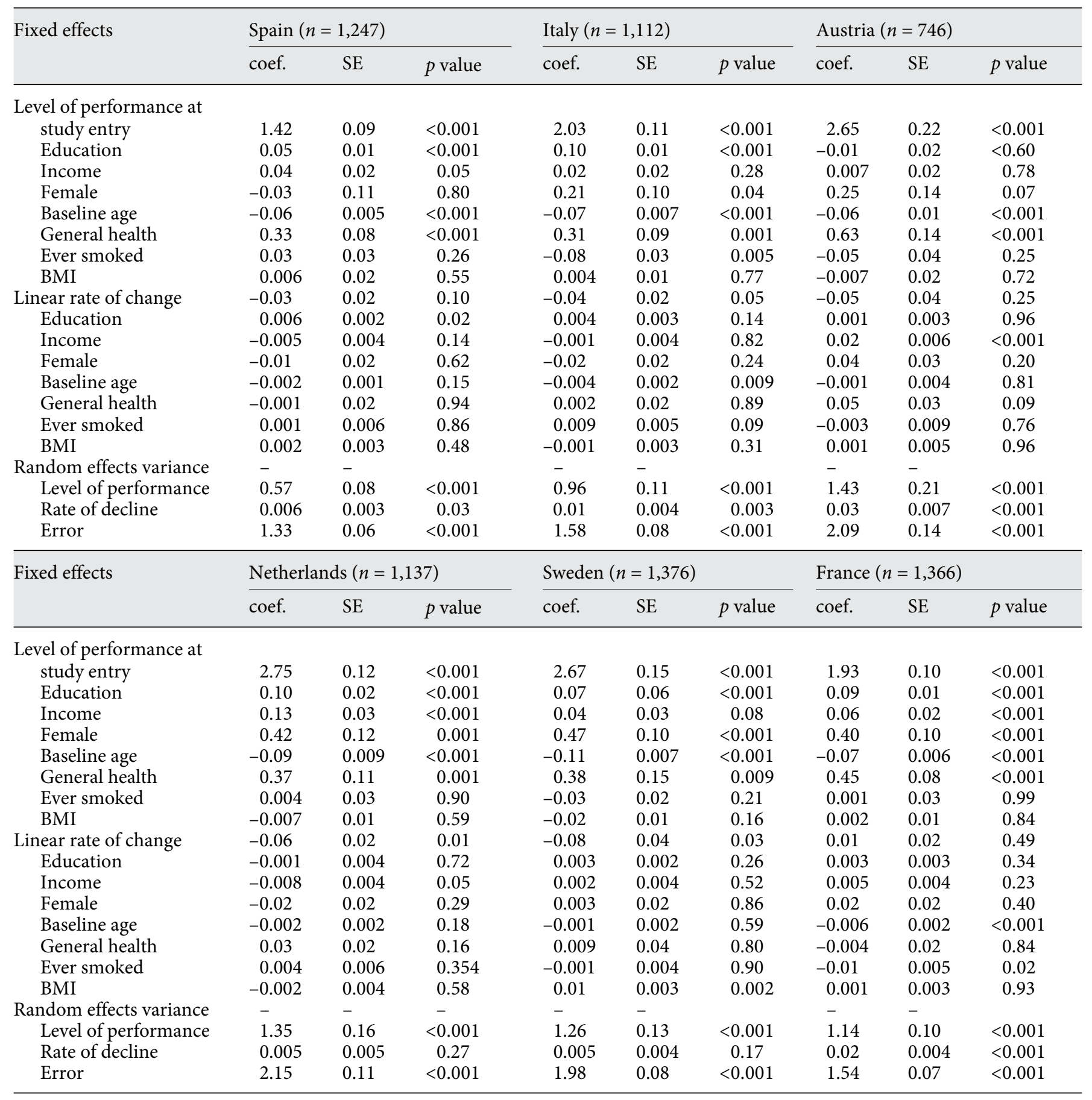

Consistently across all countries, older individuals had a worse immediate recall at study entry compared to the younger individuals. There was some evidence that they also declined at a faster rate in 4 of 10 countries (Belgium, France, Italy, and Spain).
There was a gender difference in immediate recall at study entry, with women performing better than men in most countries, except Spain, Italy, and Switzerland; but there was no gender difference in the observed rate of decline. 
Table 3. (continued)

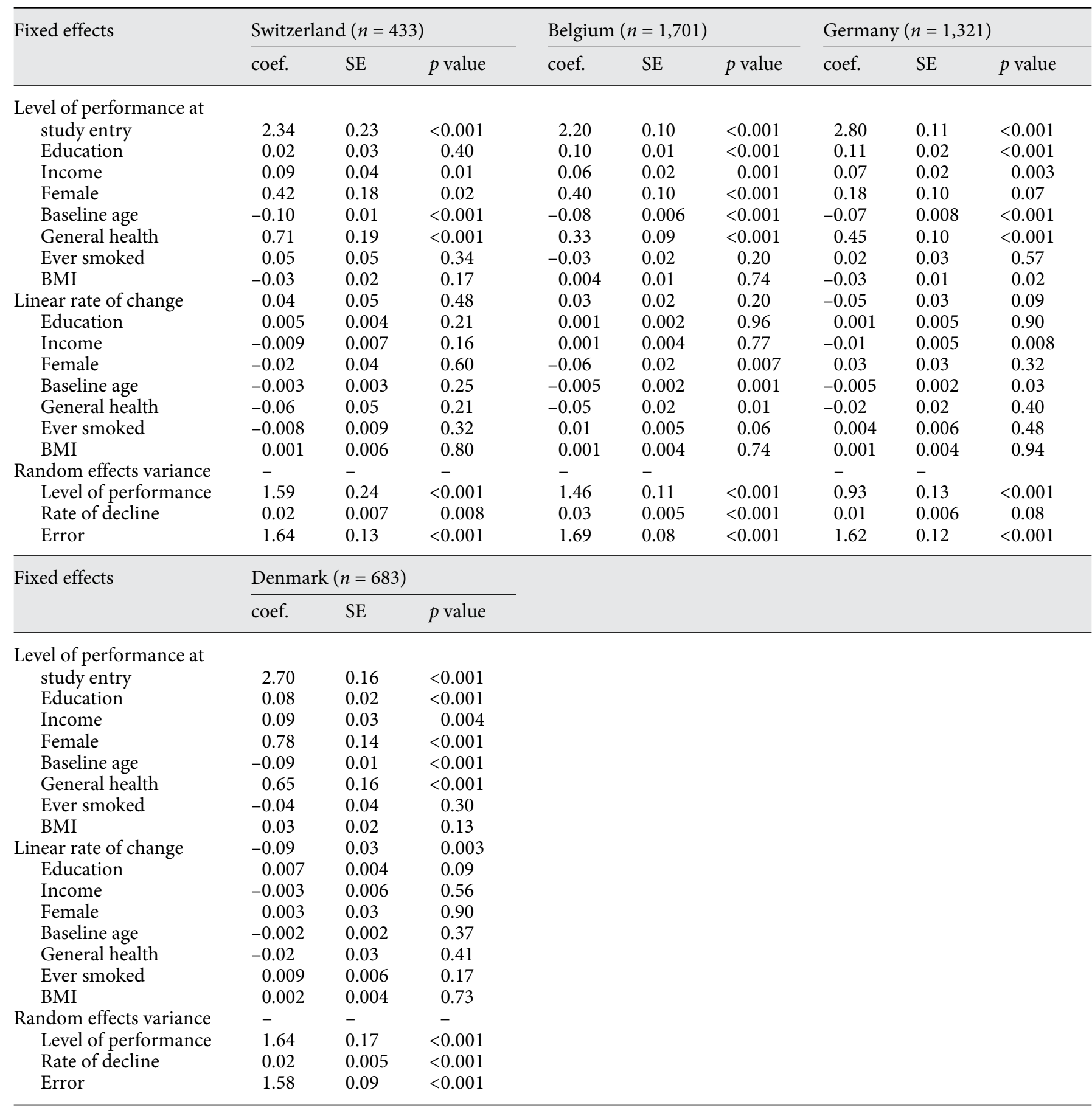

Individuals who perceived their general health to be good or excellent had better performance at study entry on immediate recall than those who perceived their health as fair or poor. Interestingly, there was no evidence that individuals who rated their health as good or excellent changed their memory performance significantly over time, compared to those who considered themselves less healthy. The only exception was Belgium, where they showed a slightly faster decline in immediate recall. 
Fig. 1. Model estimated mean trajectories of baseline performance and change over time in study (years) in immediate (red) and delayed (blue) memory recall, within each of the ten countries from SHARE. The estimates trajectories presented are for the average male participants within each country (aged 73-75, with 5-8.7 years of education and a medium gross income, who never smoked and their health was reported as fair or poor). The red trajectories represent the estimates for immediate recall and the blue ones for delayed recall. The specific colour bands represent the 95\% CIs based on the standard errors of the intercept.

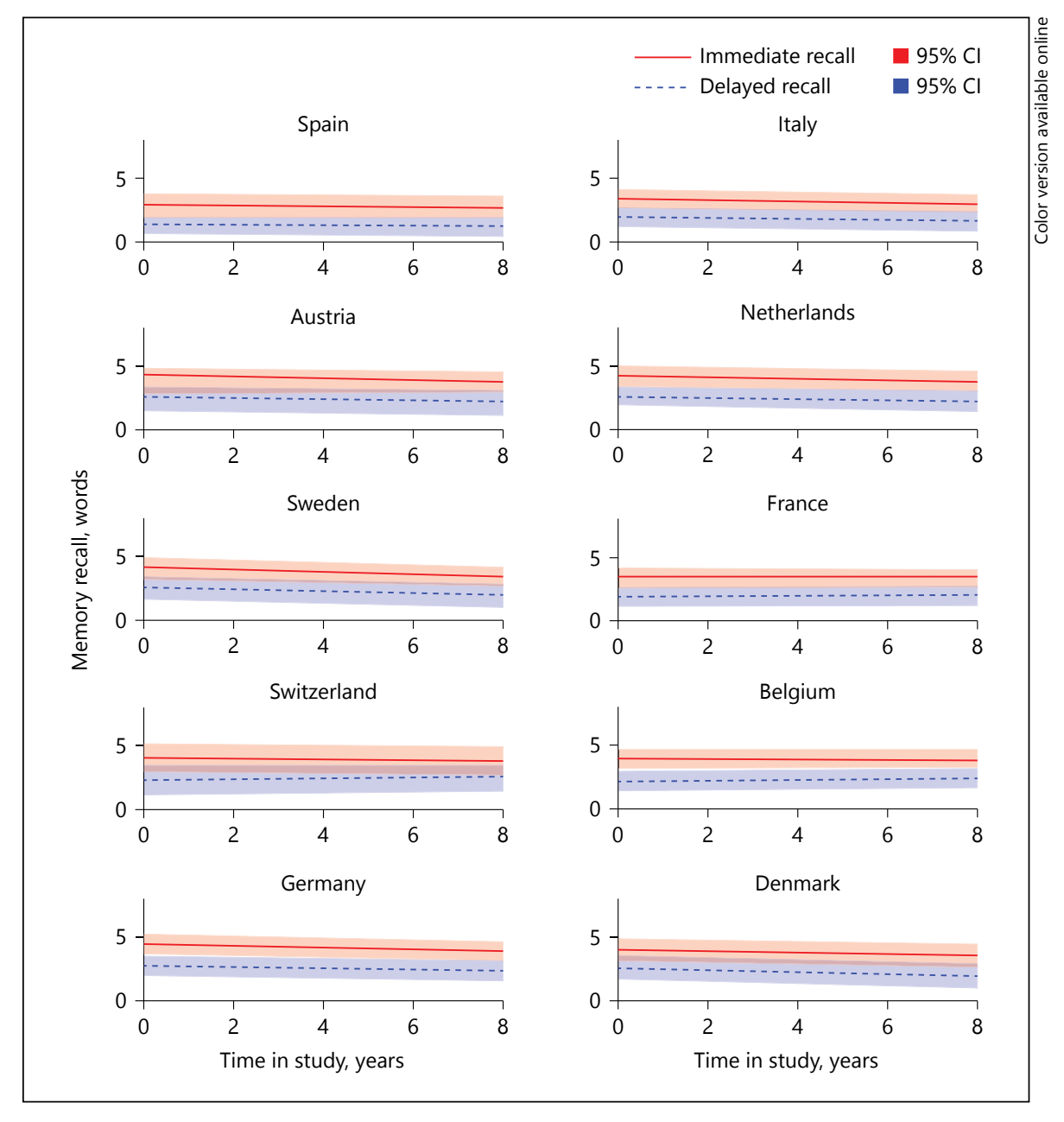

Smoking was not found to be associated with immediate memory performance or its rate of change in any of the countries, except for Italy, where smokers were found to have worse baseline performance and Spain where they declinedfasterovertime, compared to thosewhonever smoked daily. BMI was positively associated with immediate memory recall at study entry, only in one country (Denmark) and with a slower decline on the same test for Spain.

\section{The Role of Education and Other Covariates on}

Delayed Recall and Rate of Change (Table 3)

Similar to immediate recall, more educated individuals also had better performance on delayed recall at study entry compared to those with lower education. These results were consistent across 8 of 10 countries, except Austria and Switzerland. Only in Spain, education was found to be associated with the rate of change in delayed recall, where more educated individuals declined at a slower rate $(0.006[\mathrm{SE}=0.002])$.
As before, estimates of the effect of education on delayed recall at study entry and on the rate of change were also meta-analysed. The results highlight a tiny positive effect of education on the rate of change in delayed recall. The $I^{2}$ values obtained in the meta-analyses indicate a high heterogeneity between studies for the effect of education on the intercept but only low heterogeneity between studies on the estimates for the rate of change (slopes) in delayed recall (Fig. 2b, d).

Participants with higher income also performed better in the delayed memory recall performance at the study entry in most countries, with the only exceptions being Austria, Italy and Sweden. In terms of decline in this test, only wealthier nationals from Austria were protected against stronger decline, while wealthier Dutch and German nationals experienced a slightly faster decline compared to their less well-off counterparts.

Consistently with immediate recall and across all countries, older individuals also had worse performance
16

Neuroepidemiology 2017;48:9-20 DOI: $10.1159 / 000452276$
Cadar/Robitaille/Clouston/Hofer/ Piccinin/Muniz-Terrera 


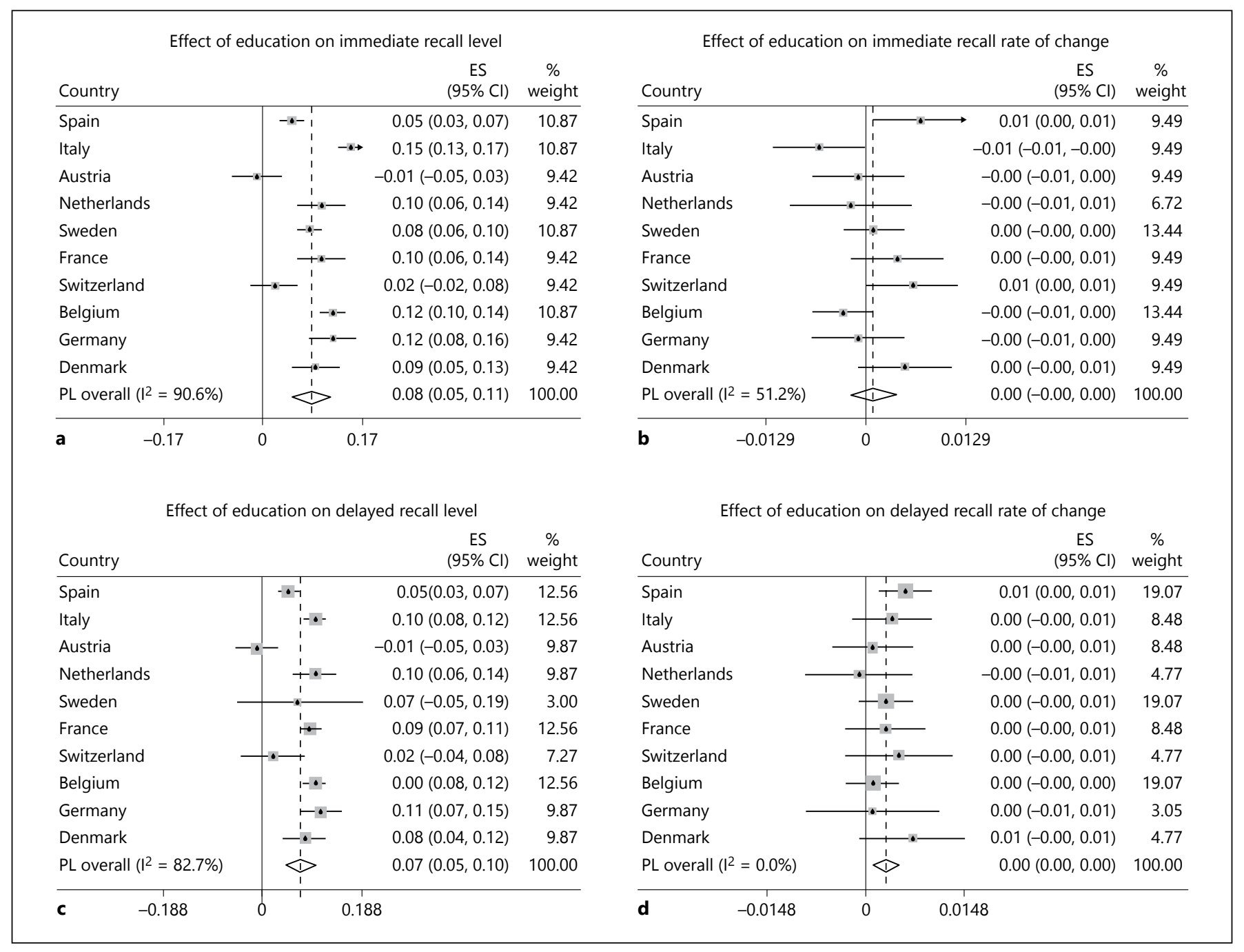

Fig. 2. Forest plots from random effects meta-analysis of estimates of the effect of education on immediate (a) and delayed (c) recall on level of performance at study entry and immediate (b) and delayed (d) rate of change.

in tests of delayed recall than younger individuals. However, the evidence that they will decline faster compared to the younger participants was observed in delayed recall only in 4 of 10 countries (Belgium, France, Italy, and Germany).

In most countries (7 out of 10$)$, there was some evidence of gender differences with women performing better than men in delayed recall at study entry. However, there were no gender differences regarding decline, except Belgium where women declined faster than men in the delayed recall.

Participants who perceived their general health to be good or excellent also had better performance at study entry on delayed memory recall in all 10 countries, compared to those who perceived their health as fair or poor.
Interestingly, there was no evidence that healthier individuals changed their memory performance significantly over time, compared to those who considered themselves less healthy, except for Belgium, where they also showed a slightly faster decline in delayed memory similar to immediate recall.

Smoking was not found to be associated with memory performance or its rate of change in any of the countries, except for Italy, where smokers were found to have worse baseline performance on both memory tests and France, where they declined slightly faster, compared to those who never smoked daily.

BMI was inversely related to performance on delayed recall at study entry in German individuals and with a slightly slower decline in participants from Sweden. 


\section{Discussion}

This study evaluated performance and rate of change in memory (immediate and delayed recall) in older individuals (aged 65 and older) in 10 European countries from SHARE. We also investigated the cognitive reserve hypothesis, operationalising reserve in terms of education, while accounting for differences in income.

Our results indicate that participants from most (6 out of 10) European countries showed a significant decline in at least one memory task (immediate recall), while 4 of these 6 countries experienced a decline in both immediate and delayed recall. Given that memory is age sensitive, these findings highlight the between-person differences reported in the literature [13] and are supported by many longitudinal studies showing subtle deterioration of memory starting as early as age 50 and decline in most other fluid cognitive abilities (e.g., attention, visuospatial ability, orientation) [14-17]. The onset of this decline could vary with age and the individual's level of education. For example, Nilsson reported an age-specific increase in semantic memory capacity up to age 55-60 years, and a significant decrease after that, especially in episodic memory as measured by free recall, cued recall, recognition and prospective memory tasks [18]. However, most elderly tend to exhibit a certain decline in fluid abilities as a result of ageing process [19], and only a lower proportion remain relatively constant or even improve their performance over time, especially in crystallised abilities such as vocabulary [13,20,21].

Our results also showed that education was associated with memory performance in both immediate and delayed recall for older Europeans, but did not show a strong moderation in the rate of change, as supported by cognitive reserve hypothesis [3, 22-24]. Similarly, in the US Health and Retirement Study (HRS), several markers of individual achievement (e.g., education, income, and wealth) were positively associated with baseline cognitive function, but not with the rate of global decline over the 12-year period [25]. This supports a larger body of literature highlighting that both high and low educated individuals decline, on average, at a similar rate $[8,14$, 26-28].

The intrinsic socioeconomic gradient for the baseline performance on immediate recall was evident in all countries investigated, despite that only wealthier individuals from one country (Austria) declined at a slower rate. Likewise, in the Maastricht Aging Study, individuals with higher professional levels showed less functional decline than their lower SES counterparts, independent of other early life influences [15]. These trends have a tendency to echo the general 'selective survival' seen in many parts of the world [16, 17].

Other modifiable risk factors such as smoking, inactivity and unhealthy diets have also been suggested to influence the rate of cognitive decline $[18,29,30]$. Several mechanisms may explain, for example, the negative impact of smoking on cognitive decline, although the precise underlying mechanism remains unclear [31]. One possibility is that increased oxidative stress is directly linked to neuronal damage [32], and smokers have been found to have reduced grey matter volume in cerebellum compared to non-smokers [33, 34]. Also, they are more likely to suffer silent infarcts or haemorrhagic strokes with direct consequences for mental function [35-37]. However, in our analyses, smoking neither influenced memory performance nor the rate of decline. Similarly, BMI showed little impact on the level of performance or the memory decline, except in Denmark where higher BMI was associated with slightly higher scores on immediate recall at baseline. However isolated, these findings mirror other reports such as HRS, where being overweight at baseline predicted better memory scores at follow-up 6-16 years later, and also testing for reverse causation; they found that preclinical dementia and cognitive impairment predicted weight loss [38].

Given the higher prevalence of illnesses associated with increasing age, controlling for health indicators in cognitive studies of ageing becomes imminent [39]. Our results showed a consistent positive association between general health and improved memory scores at baseline, but interestingly for Belgium, participants who perceived their general health to be good or excellent declined slightly faster in both immediate and delayed memory recall compared to those who evaluated their health as fair or poor. We are not sure of the nature of this isolated finding, but this inconsistency indicates a need for further investigations of health and age-related cognitive decline between different countries in Europe and across the world.

Lastly, the variation in these findings and the age of testing underscores the multi-dimensionality of the cognitive ageing process and the individual environmental influences.

\section{Strengths and Limitations}

These analyses evaluated the rate of cognitive decline in participants aged 65 years and older over an 8-year period when the accumulation of neurodegeneration starts to occur in the ageing brain but does not necessarily be-
Cadar/Robitaille/Clouston/Hofer/ Piccinin/Muniz-Terrera 
come evident. In the country level, variation in cognitive performance has rarely been investigated in healthy nonamnestic European older individuals [40]; this study makes a significant contribution to the cognitive ageing field. Furthermore, we offered an empirical contribution to the cognitive reserve hypothesis investigating both education and socioeconomic gradients in this context, in the presence of many other important modifiable risk factors such as smoking and BMI.

Despite significant differences in educational systems across countries and periods (specific laws, years of mandatory programs, fees and parental attitudes towards learning), education remains a strong indicator of cognitive function [5], but less evident for decline [15, 33], which is perhaps not necessarily reciprocally determined. We explored education as a proxy for cognitive reserve, considered to be independent of other indicators such as genetic factors $[41,42]$, childhood intelligence (IQ) or early socioeconomic influences $[4,43]$, despite a number of counterarguments suggesting that education is rather an intrinsic outcome of the level of childhood IQ and therefore closely dependent and intercalated [44-47].

Lastly, we adopted an integrative and coordinated perspective of cross-study analyses across 10 different European countries, ensuring identifiability of models that describe a linear trend of cognitive decline. This integrative approach consists of the independent but coordinated application of the same statistical model to cognitive data from each country that was amenable to longitudinal modeling, including adjustment for the same set of risk factors consistently coded across samples. Employment of such a framework facilitates the fair comparison of results as estimates of the association of risk factors with trajectory parameters have the same interpretation of studies. Another advantage is that the estimates included in the meta-analyses represent the same concept. In addition to this coordinated approach, the use of longitudinal data analysis, accounting for a broad range of factors such as gender, health, lifestyle, BMI, education, and income makes this study less susceptible to biases of non-cognitive reasons for individuals to get diagnosed within a neurological clinic, compared to others, which may help to explain divergent findings between sites with differing population protocols.

Despite these strengths, there are also several limitations. We did not account for information related to clinical diagnoses of stroke or other cardiovascular conditions. Furthermore, we need to acknowledge the drop- out at follow-up occurring in most longitudinal studies and the probability of "healthy survival" in longitudinal studies. However, the methodology employed (LGM), compensated for the missing data considered to be at random. Finally, we did not explore whether the change in general health across follow-up waves or other timevarying factors such as income, poverty, and economic hardship would have mediated the relationship between education and memory decline over time. This may represent an important direction for future research, which could be addressed with a more complex modeling approach.

\section{Conclusion}

The current analyses offered an important evaluation of the role of education on memory performance and change over time in healthy older participants educated within their country of residence, in a cross-country examination of 10 different European countries part of SHARE. Our results build on an increasingly consistent finding that education is associated with mental performance but does not seem to moderate the rate of cognitive decline.

\section{Funding and Acknowledgements}

This paper used data from SHARE Waves 1, 2, 3 (SHARELIFE), 4 and 5 (DOIs: 10.6103/SHARE.w1.260, 10.6103/SHARE.w2.260, 10.6103/SHARE.w3.100, 10.6103/SHARE.w4.111, 10.6103/ SHARE.w5.100), see Börsch-Supan et al. (2013) [48] for methodological details. The SHARE data collection has been primarily funded by the European Commission through the FP5 (QLK6CT-2001-00360), FP6 (SHARE-I3: RII-CT-2006-062193, COMPARE: CIT5-CT-2005-028857, SHARELIFE: CIT4CT-2006-028812), and FP7 (SHARE-PREP: N 211909, SHARELEAP: N 227822, SHARE M4: N 261982). Additional funding from the German Ministry of Education and Research, the US NationalInstitute on Aging(U01_AG09740-13S2,P01_AG005842, P01_AG08291, P30_AG12815, R21_AG025169, Y1-AG-4553-01, IAG_BSR06-11, OGHA_04-064) and from various national funding is gratefully acknowledged (see www.shareproject.org).

The authors of this work were supported by the following funding agencies: Alzheimer's Society (grant number 144), the Medical Research Council (programme grant number MC_UU_12019/1), and the US National Institutes of Health Unit National Institute on Aging of the National Institutes of Health under award number (grant number P01AG043362) for the Integrative Analysis of Longitudinal Studies of Aging research network. The content is solely the responsibility of the authors and does not necessarily represent the official views of the National Institutes of Health or other funding bodies mentioned above. 


\section{References}

1 Rowe JW, Kahn RL: Successful aging and disease prevention. Adv Ren Replace Ther 2000; 7:70-77.

2 Stern Y: Cognitive reserve. Neuropsychologia 2009;47:2015-2028.

3 Stern Y: What is cognitive reserve? Theory and research application of the reserve concept. J Int Neuropsychol Soc 2002;8:448-460.

4 Richards M, Sacker A: Lifetime antecedents of cognitive reserve. J Clin Exp Neuropsychol 2003;25:614-624.

5 Anstey K, Christensen H: Education, activity, health, blood pressure and apolipoprotein $\mathrm{E}$ as predictors of cognitive change in old age: a review. Gerontology 2000;46:163-177.

6 Lenehan ME, et al: Relationship between education and age-related cognitive decline: a review of recent research. Psychogeriatrics 2014, Epub ahead of print.

7 Piccinin AM, Hofer SM: Integrative analysis of longitudinal studies on aging: collaborative research networks, meta-analysis, and optimizing future studies; in Hofer SM, Alwin DF (eds): Handbook on Cognitive Aging: Interdisciplinary Perspectives. Thousand Oaks, Sage, 2008, pp 446-476.

8 Piccinin AM, et al: Coordinated analysis of age, sex, and education effects on change in MMSE scores. J Gerontol B Psychol Sci Soc Sci 2013;68:374-390.

9 Das MV, Weerman CB: Developing the survey instruments for SHARE; in Börsch-Supan A, Jürges H (eds): The Survey of Health, Ageing and Retirement in Europe - Methodology. Mannheim, MEA, 2005.

10 Muthén LK, Muthén BO: Mplus User's Guide, 2007. Los Angeles, Muthen \& Muthen.

11 StataCorp.: Stata Statistical Software: Release 13. Texas, StataCorp LP: College Station, 2013.

12 MathWorks T: MATLAB and Statistics Toolbox Release 2012b. Natick, The MathWorks, Inc., 2012.

13 Wilson RS, et al: Individual differences in rates of change in cognitive abilities of older persons. Psychol Aging 2002;17:179-193.

14 Zahodne LB, et al: Education does not slow cognitive decline with aging: 12-year evidence from the victoria longitudinal study. J Int Neuropsychol Soc 2011;17:1039-1046.

15 Bosma H, et al: To what extent does IQ 'explain' socio-economic variations in function? BMC Public Health 2007;7:179.

16 Hurst L, et al: Lifetime socioeconomic inequalities in physical and cognitive aging. Am J Public Health 2013;103:1641-1648.
17 Cagney KA, Lauderdale DS: Education, wealth, and cognitive function in later life. J Gerontol B Psychol Sci Soc Sci 2002;57:P163-P172.

18 Cadar D, et al: The role of lifestyle behaviors on 20-year cognitive decline. J Aging Res 2012;2012:304014.

19 Horn JL, Cattell RB: Age differences in fluid and crystallized intelligence. Acta Psychol (Amst) 1967;26:107-129.

20 Christensen $\mathrm{H}$ : What cognitive changes can be expected with normal ageing? Aust N Z J Psychiatry 2001;35:768-775.

21 Wilson RS, et al: Cognitive activity in older persons from a geographically defined population. J Gerontol B Psychol Sci Soc Sci 1999; 54:P155-P160.

22 Stern Y: Cognitive reserve: a catalyst for research. J Neurol Sci 2009;283:256.

23 Stern Y: Cognitive reserve in ageing and $\mathrm{Al}$ zheimer's disease. Lancet Neurol 2012;11: 1006-1012.

24 Stern Y, et al: Rate of memory decline in AD is related to education and occupation: cognitive reserve? Neurology 1999;53:1942-1947.

25 González HM, et al: What do parents have to do with my cognitive reserve? Life course perspectives on twelve-year cognitive decline. Neuroepidemiology 2013;41:101-109.

26 Ritchie SJ, et al: Education is associated with higher later life IQ scores, but not with faster cognitive processing speed. Psychol Aging 2013;28:515-521.

27 Glymour MM, Tzourio C, Dufouil C: Is cognitive aging predicted by one's own or one's parents' educational level? Results from the threecity study. Am J Epidemiol 2012;175:750-759.

28 Early DR, et al: Demographic predictors of cognitive change in ethnically diverse older persons. Psychol Aging 2013;28:633-645.

29 Durazzo TC, Mattsson N, Weiner MW; Alzheimer's Disease Neuroimaging Initiative: Smoking and increased Alzheimer's disease risk: a review of potential mechanisms. Alzheimers Dement 2014;10(3 suppl):S122S145.

30 Lehert $\mathrm{P}$, et al: Individually modifiable risk factors to ameliorate cognitive aging: a systematic review and meta-analysis. Climacteric $2015 ; 18: 678-689$.

31 Peters R, et al: Smoking, dementia and cognitive decline in the elderly, a systematic review. BMC Geriatr 2008;8:36.

32 Pimentel C, et al: Oxidative stress in Alzheimer's and Parkinson's diseases: insights from the yeast Saccharomyces cerevisiae. Oxid Med Cell Longev 2012;2012:132146.

33 Brody AL, et al: Differences between smokers and nonsmokers in regional gray matter volumes and densities. Biol Psychiatry 2004;55: $77-84$.
34 Gallinat J, et al: Smoking and structural brain deficits: a volumetric MR investigation. Eur J Neurosci 2006;24:1744-1750.

35 Longstreth WT Jr, et al: Incidence, manifestations, and predictors of worsening white matter on serial cranial magnetic resonance imaging in the elderly: the Cardiovascular Health Study. Stroke 2005;36:56-61.

36 Longstreth WT Jr: Brain abnormalities in the elderly: frequency and predictors in the United States (the Cardiovascular Health Study). Cardiovascular Health Study Collaborative Research Group. J Neural Transm Suppl 1998;53:9-16.

37 Vermeer SE, et al: Silent brain infarcts and white matter lesions increase stroke risk in the general population: the Rotterdam Scan Study. Stroke 2003;34:1126-1129.

38 Suemoto CK, et al: Body mass index and cognitive function: the potential for reverse causation. Int J Obes (Lond) 2015;39:1383-1389.

39 Fozard JL, Metter EJ, Brant LJ: Next steps in describing aging and disease in longitudinal studies. J Gerontol 1990;45:P116-P127.

40 Langa KM, et al: Cognitive health among older adults in the United States and in England. BMC Geriatr 2009;9:23.

41 Bouchard TJ Jr, McGue M: Familial studies of intelligence: a review. Science 1981;212:10551059.

42 Wilson RS, et al: The apolipoprotein E epsilon 2 allele and decline in episodic memory. J Neurol Neurosurg Psychiatry 2002;73:672-677.

43 Tucker-Drob EM, Johnson KE, Jones RN: The cognitive reserve hypothesis: a longitudinal examination of age-associated declines in reasoning and processing speed. Dev Psychol 2009;45:431-446.

44 Deary IJ, Batty GD: Commentary: pre-morbid IQ and later health - the rapidly evolving field of cognitive epidemiology. Int J Epidemiol 2006;35:670-672.

45 Deary IJ, Batty GD: Cognitive epidemiology. J Epidemiol Community Health 2007;61: 378-384

46 Batty GD, Deary IJ, Macintyre S: Childhood IQ in relation to risk factors for premature mortality in middle-aged persons: the Aberdeen Children of the 1950s study. J Epidemiol Community Health 2007;61:241-247.

47 Deary IJ, et al: Age-associated cognitive decline. Br Med Bull 2009;92:135-152.

48 Börsch-Supan A, et al: Data resource profile: the survey of health, ageing and retirement in Europe (SHARE). Int J Epidemiol 2013;42: 992-1001. 